\title{
A New Thin Endoscopic Method of Transanal Drainage Tube Insertion for Acute Colonic
}

\section{Obstruction Due to Colorectal Cancer}

Ken Ohnita ${ }^{1}$, Saburo Shikuwa ${ }^{1}$, Hajime Isomoto ${ }^{1}$, Naoyuki Yamaguchi ${ }^{1}$, Kenta Okamoto ${ }^{2}$, Hitoshi Nishiyama $^{2}$, Eiichiro Fukuda ${ }^{2}$, Takashi Nakamura ${ }^{2}$, Yohei Mizuta ${ }^{1}$, Shigeru Kohno ${ }^{1}$

${ }^{1}$ Second Department of Internal Medicine, Nagasaki University School of Medicine, Nagasaki, Japan

${ }^{2}$ Department of Internal Medicine, Nagasaki National Medical Center, Nagasaki, Japan

Running title: transanal drainage by thin endoscope

Address for correspondence and reprint requests: Ken Ohnita, MD

Second Department of Internal Medicine, Nagasaki University School of Medicine, 1-7-1 Sakamoto,

Nagasaki 852-8501, Japan

E-mail: k-ohnita@nagasaki-u.ac.jp

Telephone: +81-95-819-7273, Fax: +81-95-849-7285 


\section{Abstract}

Aim: Insertion of a transanal drainage tube is effective in the management of obstructing colorectal cancer.

We devised a new method of inserting the transanal drainage tube safely and quickly using a thin

endoscope.

Methods: Sixteen patients (7 men and 9 women) with obstructive left-sided colorectal cancer were treated

by inserting a transanal drainage tube using a thin endoscope. We inserted a transanal drainage tube for 32

patients (21 men and 11 women) of left-sided colorectal cancer by conventional method.

Results: Drainage tube placement by conventional method was successful in 29 (90.6\%) of 32 patients,

while, via new method, it was successful in all 16 patients without major complications. Moreover,

median insertion time was significantly shortened (34 minutes for the new method compared with 42

minutes for the conventional method).

Conclusion: Management of acute colorectal obstruction by transanal drainage tube insertion using the thin endoscope was effective and safe.

Keywords:

transanal drainage tube, thin endoscope, obstructive colorectal cancer 


\section{Introduction}

Obstructive colorectal cancer needs immediate treatment. Although colostomy is useful, it requires two-step surgery and is a costly and time-consuming procedure. One-step surgery, on the other hand, often causes postoperative complications such as leakage, postoperative stenosis, and poor general condition, resulting in high rates of morbidity and mortality ${ }^{1}$.

Recently, regardless of the site of colorectal obstruction, the development of a new transanal drainage tube and techniques for its use has made it possible to decompress acute colorectal obstruction ${ }^{2-4}$. Several advantages of this method have been reported. However, Sumiyama et al. reported that insertion of a transanal drainage tube was complicated by guide wire perforation in $0-6 \%$ of cases ${ }^{5}$.

We devised a new method of safely inserting a transanal drainage tube using a thin endoscope and herein report this method together with a comparison with the conventional method.

\section{Patients and Methods}

\section{Patients}

Between September 2002 and October 2005, we inserted a transanal drainage tube for 32 patients of left-sided colon cancer by conventional method (21 men and 11 women, aged 49-88 years, mean=69 years) with obstructive left-sided colorectal cancer (Table 1). Between November 2005 and May 2008, 
we inserted a transanal drainage tube using a thin endoscope for 16 patients ( 7 men and 9 women, aged 31-95 years, mean=68 years) with obstructive left-sided colorectal cancer (Table 1). Obstructive colorectal cancers were diagnosed by the existence of air-fluid level and dilatation of the colon proximal to the tumors in the abdominal X-ray and CT scan. There was no statistical difference in the location sites of the lesions between the conventional and new method (Table 1). The study was conducted in accordance with the declaration of Helsinki. Written informed consent was obtained in each patient.

Drainage tube

We used a 120-cm 22-F Dennis Colorectal Tube (Nippon Sherwood, Tokyo, Japan).

Conventional method

Glycerol enema was performed before the procedure. The endoscope (Olympus CF-Q240 or Olympus CF-Q260AI; Olympus Co., Ltd., Tokyo, Japan) was inserted through the anus to the anal side of the tumor. Through the endoscope, a 0.052 inch guide wire was inserted through the narrowed lumen to the oral side of the lesion. The endoscope was removed, and the Dennis colorectal drainage tube was introduced over the guide wire. After confirming that the balloon part of the tube had passed the lesion, the balloon was inflated with sterilized water $(15-20 \mathrm{ml})$, and the position of the tube was fixed. Finally, the intestinal tract 
was flushed several times using $500 \mathrm{ml}$ of water.

New method (Figure 1,2)

Glycerol enema was performed before the procedure. The endoscope (Olympus GIF-N230: $6 \mathrm{~mm}$ diameter; Olympus Co., Ltd., Tokyo, Japan) was inserted from the anal side of the tumor through the stenosis to the oral side of the tumor. Through the endoscope, a 0.052 inch guide wire was inserted. The endoscope was removed, and the Dennis colorectal drainage tube was introduced over the guide wire. After confirming that the balloon part of the tube had passed the lesion, the balloon was inflated with sterilized water $(15-20 \mathrm{ml})$, and the position of the tube was fixed. Finally, the intestinal tract was flushed several times using $500 \mathrm{ml}$ of water.

In both methods, the intestinal tract was cleaned two or three times daily using 500 to $1000 \mathrm{ml}$ of water for a few days before operation until there was no fecal colonic content.

\section{Results}

Results with the conventional method and the new method are compared in Table 1. Drainage tube placement was successful in 29 (90.6\%) of 32 patients in conventional method; it was not possible to pass 
the guide wire through the obstructive segment in three patients. In one of the unsuccessful cases, the patient went into shock and had to undergo urgent operation. On the other hand, drainage tube placement was successful in all 16 patients with the new method, and there were no major complications that needed treatment. Moreover, Median insertion time was significantly shortened (42 minutes for the conventional method vs. 34 minutes for the new method, $\mathrm{p}<0.05$ by Mann-Whitney' $\mathrm{U}$ test).

\section{Discussion}

Acute colorectal obstruction by colorectal carcinoma is an emergency situation that necessitates immediate surgical treatment and has a poor prognosis ${ }^{6}$. As a preoperative treatment, the usefulness of endoscopic decompression using self-expandable metallic stents has been reported ${ }^{7-9}$. However, placing a drainage tube is easier than introducing a metallic stent, with a lower complication rate during and after the procedure. In addition, drainage tubes are less expensive than expandable metallic stents ${ }^{3}$, and they can not only decompress the intestinal tract but also examine the oral side of the tumor by enabling gastrografin enema. On the other hand, the rate of successfully placing a drainage tube varies from $60-100 \%$ depending on the operators ${ }^{5}$. Moreover, the rate of guide wire perforation is $0-6 \%$ at the time of tube insertion ${ }^{5}$.

In the present study, we evaluated drainage tube insertion using a thin endoscope. The rate of 
successful insertion in the new method was excellent with $100 \%(16 / 16)$. On the other hand, with conventional method, the rate of successful insertion in the conventional method was $90.6 \%$ (29/32). In the conventional method, insertion failure resulted from being unable to pass the guide wire through the stenosis.

The Olympus GIF-N230 scope is marketed as a transnasal endoscope. Although it has poorer image quality than standard endoscopes it has the advantage of a small diameter (about $6 \mathrm{~mm}$ ). We therefore used this scope to insert the transanal drainage tube.

A thin endoscope has several advantages in the current situation. It is relatively soft and can easily be used to inspect stenosis, even in tortuous regions of the sigmoid colon. It avoids the risk of perforation associated with passing a guide wire through the stenosis. Of note, the new method significantly reduced median insertion time from 42 minutes (for the conventional method) to 34 minutes. In this study, since the stage when each procedure was performed differed between the conventional method and the new method, the experience for each method might influence on the results. Considering the location of tumors and the degree of stenosis varied, the results should be interpreted carefully. Nevertheless, results using the thin endoscope seem to be less dependent on the operator's degree of skill than those with the conventional endoscope. On the other hand, since the thin scope is soft, the insertion to the transverse, ascending colon and caecum is relatively difficult. In such cases, we are to insert the 
drainage tube by conventional method. Moreover, it may be sometimes difficult to put in a scope for the elongated sigmoid colon. In such cases, we are usually press the abdomen by an assistant to help the insertion. Moreover, it is sometimes difficult to pass a scope for marked stenosis. In fact, the diameter of thin scope is thinner than the drainage tube, and we could insert the scope into the narrowed segments in all the present cases. However, it may be necessary to use a dilator following lefting a guide wire, in cases of colorectal cancers with severe stenosis through which even the thin scope cannot pass.

The limitation of this study is retrospective; obviously, a larger prospective radomized trial are warrant to confirm the efficacy of this new strategy for acute colonic obstruction due to colorectal malignancies.

In conclusion, preoperative management of acute colorectal obstruction by insertion of transanal drainage tube using a thin endoscope was effective and safe. 


\section{References}

1. Deans GT, Krukowski ZH, Irwin ST. Malignant obstruction of the left colon. Br J Surg. 1994; 81: 1270-6

2. Nozoe T, Matsumata T. Usefulness of preoperative colonic lavage using transanal ileus tube for obstructing carcinoma of left colon. J Clin Gastroenterol. 2000; 31(2): 156-8.

3. Tanaka T, Furukawa A, Murata K, et al. Endoscopic transanal decompression with a drainage tube for acute colonic obstruction. Dis Colon Rectum. 2001; 44(3): 418-22.

4. Horinouchi A, Nakayama Y, Tanaka N, et al. Acute colorectal obstruction treated by means of transanal drainage tube: Effectiveness before surgery and stenting. Am J Gastroenterol. 2005; 100: 2765-70.

5. Sumiyama Y, Saida Y. Stent endoprosthesis and transanal decompression tube for colorectal obstructions. Clinical Gastroenterology (in Japanese). 2005; 20(13): 1777-84.

6. Umpleby HC, Williamson RC. Survival in acute obstructing colorectal carcinoma. Dis Colon Rectum. 1984; 27: 299-304.

7. Khot UP, Lang AW, Murali K, et al. Systemic review of the efficacy and safety of colorectal stents. Br J Surg. 2002; 89: 1096-102.

8. Baron TH, Harewood GC. Enteral self-expandable stents. Gastrointest Endosc. 2003; 58: 421-33. 
9. Suzuki N, Saunders BP, Thomas-Gibson S, et al. Colorectal stenting for malignant and benign disease: Outcomes in colorectal stenting. Dis Colon Rectum. 2004; 47: 1201-7. 
Figure legends

Figure 1

A. The endoscope was passed through the stenosis to the oral side of the tumor. A guide wire was then inserted through the endoscope.

B. After the scope was removed, the drainage tube was inserted through the lesion over the guide wire.

C. The balloon was inflated, fixing the tube in place.

Figure 2

A. A tumor was recognized in the sigmoid colon.

B. A thin endoscope was inserted into the stenosis. 
A

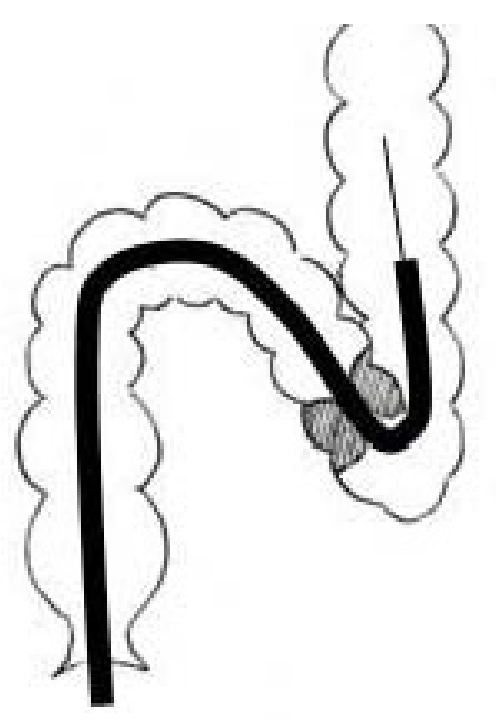

B

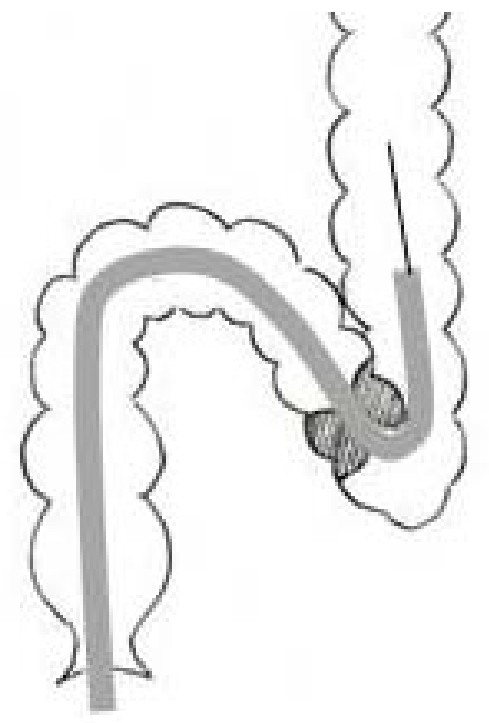

C

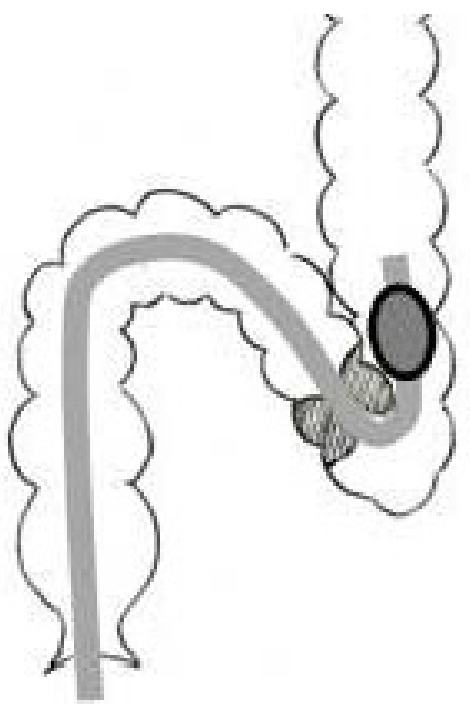

Figure 1 


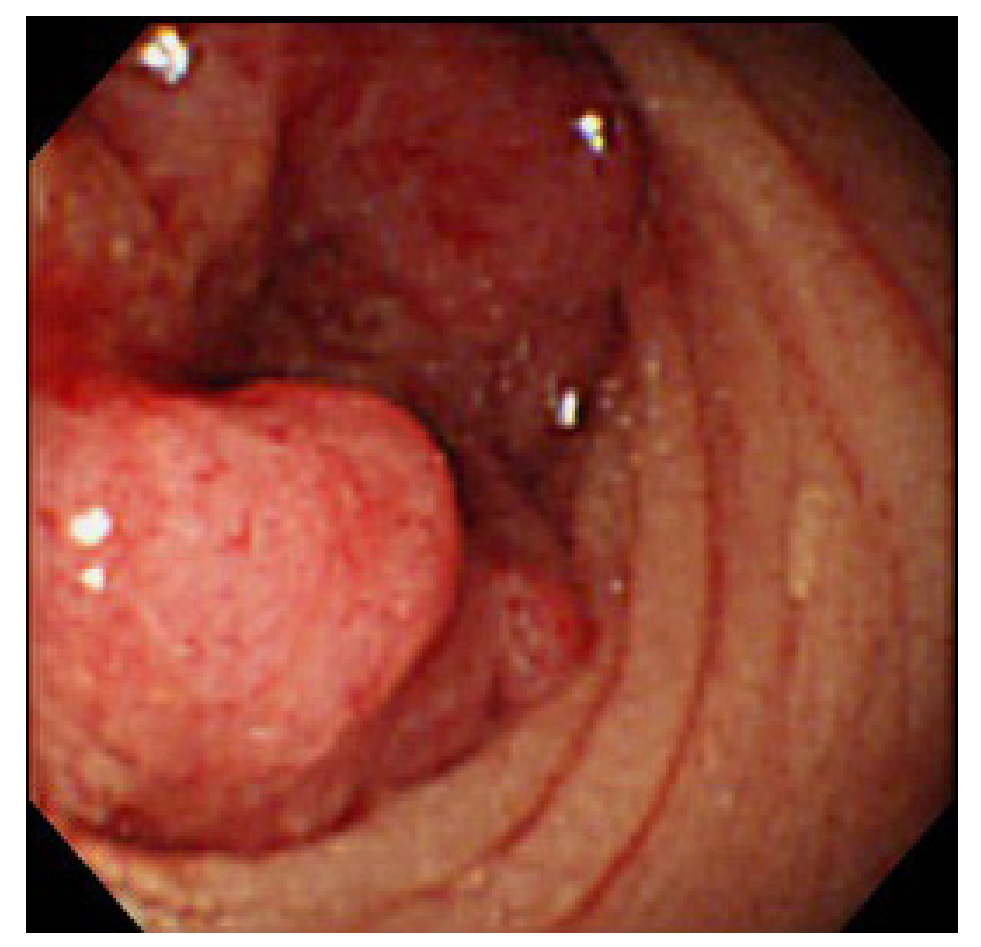

Figure 2A 


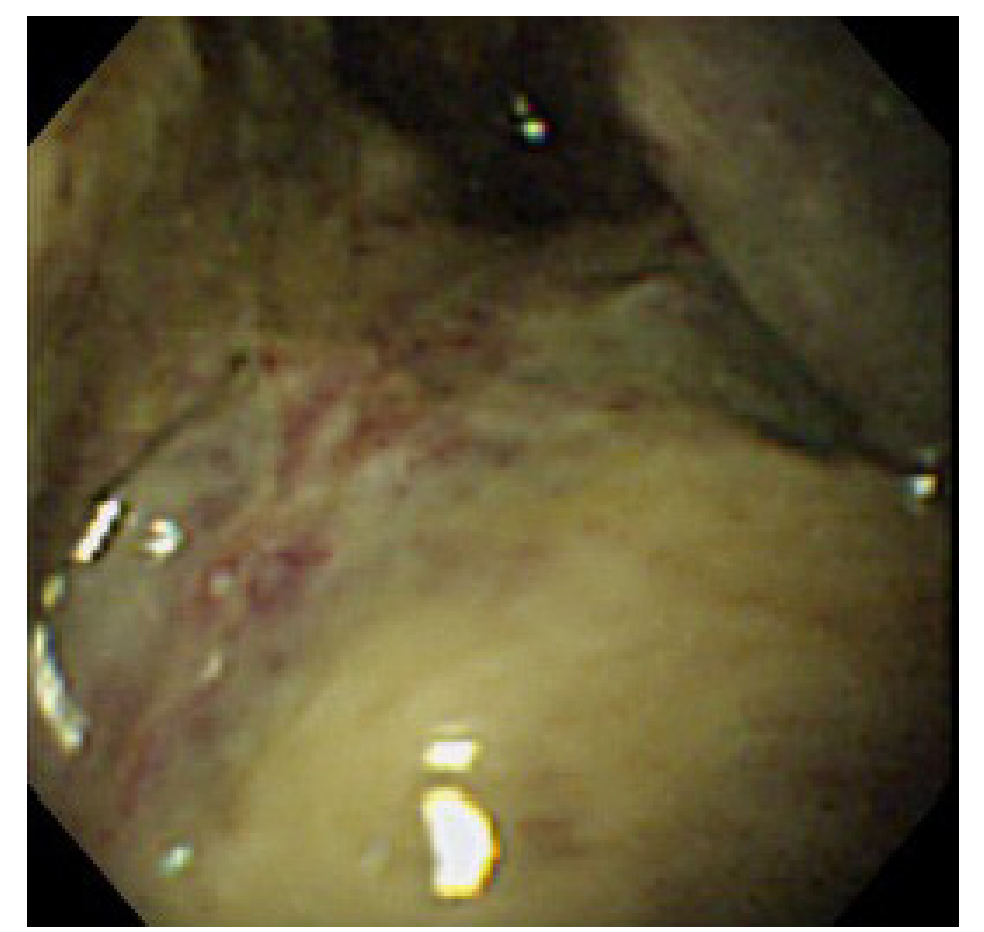

Figure 2B 


\begin{tabular}{|l|c|c|}
\hline & $\begin{array}{c}\text { Conventional } \\
\text { method } \\
(\mathrm{n}=32)\end{array}$ & New method \\
$(\mathrm{n}=16)$
\end{tabular}

Table 1

\# R: rectum

* Median

S: sigmoid colon

D: descending colon 